\title{
Quantifying Political Leaning from Tweets, Retweets, and Retweeters
}

\author{
Felix Ming Fai Wong, Student Member, IEEE, Chee Wei Tan, Senior Member, IEEE, Soumya Sen, Mung \\ Chiang, Fellow, IEEE
}

\begin{abstract}
The widespread use of online social networks (OSNs) to disseminate information and exchange opinions, by the general public, news media and political actors alike, has enabled new avenues of research in computational political science. In this paper, we study the problem of quantifying and inferring the political leaning of Twitter users. We formulate political leaning inference as a convex optimization problem that incorporates two ideas: (a) users are consistent in their actions of tweeting and retweeting about political issues, and (b) similar users tend to be retweeted by similar audience. Then for evaluation and a numerical study, we apply our inference technique to 119 million election-related tweets collected in seven months during the 2012 U.S. presidential election campaign. Our technique achieves $94 \%$ accuracy and high rank correlation as compared with manually created labels. By studying the political leaning of 1,000 frequently retweeted sources, 230,000 ordinary users who retweeted them, and the hashtags used by these sources, our numerical study sheds light on the political demographics of the Twitter population, and the temporal dynamics of political polarization as events unfold.
\end{abstract}

Index Terms-Twitter, political science, political polarization, data analytics, inference, convex optimization, regularization

\section{INTRODUCTION}

$I^{\mathrm{N}}$ $\mathrm{N}$ recent years, big online social media data have found many applications in the intersection of political and computer science. Examples include answering questions in political and social science (e.g., proving/disproving the existence of media bias $[3,24]$ and the "echo chamber" effect $[1,5])$, using online social media to predict election outcomes [37, 25], and personalizing social media feeds so as to provide a fair and balanced view of people's opinions on controversial issues [30]. A prerequisite for answering the above research questions is the ability to accurately estimate the political leaning of the population involved. If it is not met, then for the above examples, either the conclusion will be invalid, the prediction will perform poorly $[29,31]$ due to a skew towards highly vocal individuals [27], or user experience will suffer.

In the context of Twitter, accurate political leaning estimation poses two key challenges: (a) Quantification: Is it possible to assign meaningful numerical scores to tweeters about their position in the political spectrum? (b) Scalability: Given Twitter's large scale and server limitations, how can we devise a method that is efficient and scalable? We propose a new approach that incorporates the following two sets of information to infer the political leaning of a target set of Twitter users.

- Felix M.F. Wong and Mung Chiang are with the Department of Electrical Engineering, Princeton University. Email: \{mwthree, chiangm\}@princeton.edu

- Chee Wei Tan is with the Department of Computer Science, City University of Hong Kong. Email: cheewtan@cityu.edu.hk

- Soumya Sen is with the Department of Information Systems \& Decision Sciences, University of Minnesota. Email: ssen@umn.edu

Preliminary version in [42]. This version has substantial improvements in algorithm, evaluation and numerical studies.
Tweets and retweets: the target users' temporal patterns of being retweeted, and the tweets published by their retweeters. The insight is that a user's tweet contents should be consistent with who they retweet, e.g., if a user tweets a lot during a political event, she is expected to also retweet a lot at the same time. This is the "time series" aspect of the data.

Retweeters: the identities of the users who retweeted the target users. The insight is similar users get followed and retweeted by similar audience due to the homophily principle. This is the "network" aspect of the data.

Our technical contribution is to frame political leaning inference as a convex optimization problem that jointly maximizes tweet-retweet agreement with an error term, and user similarity agreement with a regularization term which is constructed to also account for heterogeneity in data. The result is an inference technique that is:

- Scalable: it does not require explicit knowledge of the network topology, and works within rate limits imposed by the Twitter API;

- Efficient: computationally efficient because it is formulated as a convex optimization problem, and data efficient because the time required to collect sufficient data to obtain good results is short; and

- Intuitive: the computed scores have a simple interpretation of "averaging," i.e., a score is the average number of positive/negative tweets expressed when retweeting the target user. See Figure 1 for an illustration.

Using a set of 119 million tweets on the U.S. presidental election of 2012 collected over seven months, we extensively evaluate our method to show that it outperforms several standard algorithms and is robust with respect to variations to the algorithm. 


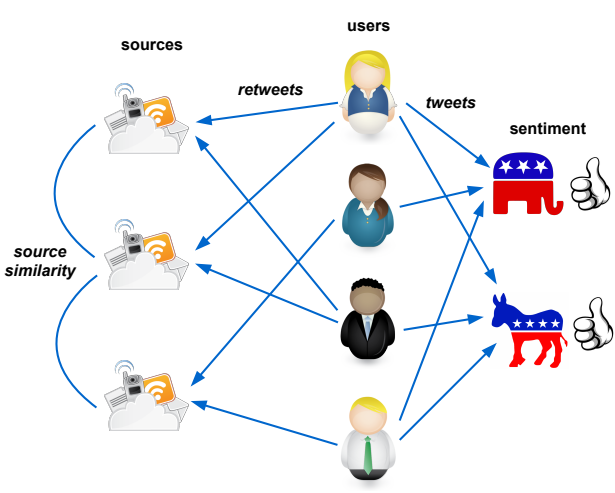

Fig. 1. Incorporating tweets and retweets to quantify political leaning.

The second part of this paper presents a numerical study on our collected tweets from the 2012 election, by first (a) quantifying the political leaning of 1,000 frequentlyretweeted Twitter users, and then (b) using their political leaning, infer the leaning of 232,000 ordinary Twitter users. We make a number of findings:

- Parody Twitter accounts have a higher tendency to be liberal as compared to other account types. They also tend to be temporally less stable.

- Liberals dominate the population of less vocal Twitter users with less retweet activity, but for highly vocal populations, the liberal-convservative split is balanced. Partisanship also increases with vocalness of the population.

- Hashtag usage patterns change significantly as political events unfold.

- As an event is happening, the influx of Twitter users participating in the discussion makes the active population more liberal and less polarized.

The organization of the rest of this paper is as follows. Section 2 reviews related work in studies of Twitter and quantifying political orientation in traditional and online social media. Section 3 details our inference technique by formulating political leaning inference as an optimization problem. Section 4 describes our dataset collected during the U.S. presidential election of 2012, which we use to derive ground truth for evaluation in Section 5. Then in Section 6 we perform a numerical study on the same dataset, studying the political leaning of Twitter users and hashtags, and how it changes with time. Section 7 concludes the paper with future work.

\section{Related Work}

In political science, the ideal point estimation problem $[34,8]$ and its Bayesian extensions [17, 18] aim to estimate the political leaning of legislators from roll call data. This line of work assumes legislators to vote probabilistically according to their positions ("ideal points") in a latent space, and the latent positions are statistically inferred from observed data, i.e., how they vote. The main difference between our work and this line of work is in the data: while legislators are characterized by their voting history, which can be considered as their explicit stances on various issues, we do not have access to comparably detailed data for most Twitter users.

A variety of methods have been proposed to quantify the extent of bias in traditional news media. Indirect methods involve linking media outlets to reference points with known political positions. For example, Lott and Hassett [26] linked the sentiment of newspaper headlines to economic indicators. Groseclose and Milyo [20] linked media outlets to Congress members by co-citation of think tanks, and then assigned political bias scores to media outlets based on the Americans for Democratic Action (ADA) scores of Congress members. Gentzkow and Shapiro [16] performed an automated analysis of text content in newspaper articles, and quantified media slant as the tendency of a newspaper to use phrases more commonly used by Republican or Democrat members of the Congress. In contrast, direct methods quantify media bias by analyzing news content for explicit (dis)approval of political parties and issues. Ho and Quinn [21] analyzed newspaper editorials on Supreme Court cases to infer the political positions of major newspapers. Ansolabehere et al. [4] used 60 years of editorial election endorsements to identify a gradual shift in newspapers' political preferences with time.

Except for [16], the above studies require some form of manual coding and analysis, which is expensive and timeconsuming. A more fundamental problem is data scarcity. Because the amount of data available for analysis is limited by how fast the media sources publish, researchers may need to aggregate data created over long periods of time, often years, to perform reliable analysis. Analyzing media sources through their OSN outlets offers many unprecedented opportunities with high volume data from interaction with their audience.

Political polarization has been studied in different types of online social media. Outside of Twitter, Adamic and Glance [1] analyzed link structure to uncover polarization of the political blogosphere. Zhou et al. [43] incorporated user voting data into random walk-based algorithms to classify users and news articles in a social news aggregator. Park et al. [32] inferred the political orientation of news stories by the sentiment of user comments in an online news portal. Weber et al. [40] assigned political leanings to search engine queries by linking them with political blogs. Regarding Twitter, political polarization was studied in [11]. Machine learning techniques have been proposed to classify Twitter users using e.g., linguistic content, mention/retweet behavior and social network structure $[6,2,33,9]$. Conover et al. [10] applied label propagation to a retweet graph for user classification, and found the approach to outperform tweet content-based machine learning methods.

Our problem of assigning meaningful political leaning scores to Twitter users is arguably more challenging than the above classification problem. There have already been several works on quantifying political leaning using the Twitter follower network. An et al. [3] and King et al. [22] applied multidimensional scaling on media sources with their pairwise distances computed from their mutual follower sets. Barberá [5] applied Bayesian ideal point estimation using following actions as observations. Golbeck and Hansen [19] proposed a graph-based method to propagate ADA scores of Congress members on Twitter to media sources through 
their followers. Weber et al. [41] quantified the political leaning of Twitter hashtags.

We argue that using retweet, rather than follower, data has its advantages. First, the huge sizes of most OSNs mean it is difficult for an average researcher to obtain an upto-date snapshot of a network. The Twitter API prevents crawling the network beyond the one-hop neighborhood of a few thousand nodes. ${ }^{1}$ On the other hand, our method requires only one connection to the real-time Twitter stream to collect retweets. Second, retweet data is more robust than follower data. Retweeting is an explicit act of approval, but following is not, e.g., a user can follow two users with opposing stances to get a balanced view. Also, the follower graph is static in the sense that it does not capture real-time information flow and lacks fine-grained edge creation times.

Besides [10], retweet data have been applied in several recent works. Our retweet-based regularization is related to [13], which built a co-retweeted network for studying political polarization, and [39], which proposed a regularization framework using co-retweeting and co-retweeted information. Volkova et al. [38] built a series of Twitter social graphs to augment neighbors features (also studied in [2] but not on retweet graphs) to improve performance. Compared to the above, our work (a) does not directly use a co-retweeted network but adds a matrix scaling preprocessing step to account for heterogeneity in Twitter users' popularity, (b) introduces the tweet-retweet consistency condition, and (c) performs a longitudinal study on our dataset collected over seven months.

\section{FORMULATION}

\subsection{Motivation and Summary}

To motivate our approach in using retweets for political leaning inference, we present two examples to highlight the existence of useful signals from retweet information.

From our dataset on the 2012 presidential election (see details in Section 4), we identify the Twitter accounts of two major media sources, one with liberal- and the other with conservative-leaning. In Figure 2 we plot their retweet popularity (their columns in matrix $\mathbf{A}$, see Section 3.2) during the 12 events in the dataset (see Table 1). We observe negative correlation between two sources' patterns of being retweeted, especially during events 6 and $7 .^{2}$ This can be explained by Democrat/Republican supporters enthusiastically retweeting Romney/Obama-bashing tweets published by the media outlets during the corresponding events.

This example provides two hints: (a) The number of retweets received by a retweet source during an event can be a signal of her political leaning. In particular, one would expect a politically inclined tweeter to receive more retweets during a favorable event. (b) The action of retweeting carries implicit sentiment of the retweeter. This is true even if

1. As of the time of writing, each authenticated client can make 350 requests/hour, with each request returning at most 5,000 followers of a queried user. This is not to say scraping the complete network is impossible, but many tricks are needed [15].

2. Event 6: a video was leaked with Romney criticizing " $47 \%$ of Americans [who pay no income tax]" at a private fundraiser. Event 7: the first presidential debate, where Obama was criticized for his poor performance.

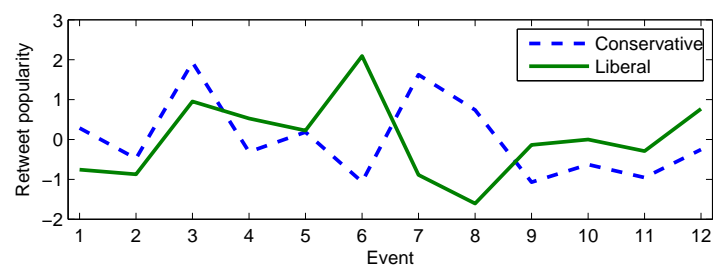

Fig. 2. Negatively correlated retweet patterns of two media Twitter accounts with opposite political leaning.

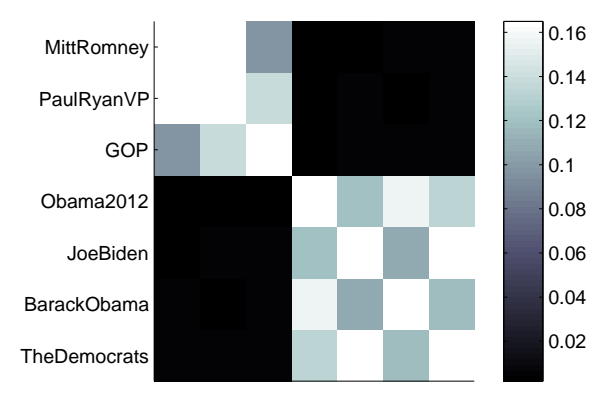

Fig. 3. Similarity of U.S. presidential election candidates by co-retweeter information. Clear separation is observed, with low similarity (dark areas) between two accounts in different parties.

the original tweet does not carry any sentiment itself. The intuition is that users tend to follow and retweet those who share similar political views, e.g., a user is more likely to retweet a newspaper to which it subscribes than any random newspaper, a manifestation of the homophily principle.

Our second example shows the identities of retweeters are a signal of one's political leaning. In Figure 3, we plot a portion of matrix $\mathbf{S}$, which stores the cosine similarity of any two Twitter accounts based on the overlap of their sets of retweeters (see Section 3.4 for details). By focusing on the election candidates ${ }^{3}$ and official political party accounts, we see a clear separation of the two camps: two same-camp accounts have similarity that is at least 14 times of that between two different-camp accounts.

Given retweet and retweeter information are useful for inferring a Twitter account's political leaning, we formulate inference as a graph Laplacian-regularized least squares problem (Section 3.5) which consists of two steps. First, we assume that there is a large Twitter population that tweet and retweet at the same time, and the two forms of expressing political opinions are consistent. Then we frame political leaning estimation as a least squares (or linear inverse) problem in Section 3.3. Second, we add a regularization term to the least squares problem to ensure similar Twitter users, i.e., those having similar sets of audience who have retweeted them, have similar political leaning. We remark that naively building the regularization matrix results in poor performance. See Section 3.4 for how we carefully construct the matrix.

3. Obama2012 is Barack Obama's official campaign account, and BarackObama is his personal account. There is no such distinction for Mitt Romney's Twitter account(s). 


\subsection{Definitions}

Consider two political parties running for an election. During the election campaign there have been $E$ events which attracted considerable attention in Twitter. We are interested in quantifying the liberal-conservative $e^{4}$ political leaning of $N$ prominent retweet sources, e.g., media outlets' Twitter accounts and celebrities.

For event $i$, let $U_{i}$ be the set of users who tweeted about the event, and $T_{i u}$ be the set of tweets sent by user $u \in U_{i}$ about the event. Also define each tweet $t$ to carry a score $s_{t} \in[-1,1]$, such that it is 1 if the tweet shows full support for one candidate, or -1 if full support is for the other. Then for user $u$ her approval score is

$$
\sum_{t \in T_{i u}} \frac{s_{t}}{\left|T_{i u}\right|}
$$

Averaging over all users in $U_{i}$, the average tweet leaning $y_{i}$ of event $i$ is ${ }^{5}$

$$
y_{i}=\frac{1}{\left|U_{i}\right|} \sum_{u \in U_{i}} \sum_{t \in T_{i u}} \frac{s_{t}}{\left|T_{i u}\right|} .
$$

For source $j$, we quantify her political leaning as ${ }^{6} x_{j} \in$ $\mathbb{R}$, interpreted as the average approval shown when someone retweets a tweet originating from $j$.

Now let $V_{i}$ be the set of users who retweeted any one of the $N$ sources during event $i^{7}$ and $R_{u j}^{(i)}$ be the number of retweets sent by user $u$ with the tweet originating from source $j$. Then the retweet approval score of user $u \in V_{i}$ is the average over all sources it has retweeted:

$$
\sum_{j=1}^{N} \frac{R_{u j}^{(i)}}{\sum_{k=1}^{N} R_{u k}^{(i)}} x_{j}
$$

and the average retweet leaning is the average over all $u$ :

$$
\begin{aligned}
& \frac{1}{\left|V_{i}\right|} \sum_{u \in V_{i}} \sum_{j=1}^{N} \frac{R_{u j}^{(i)}}{\sum_{k=1}^{N} R_{u k}^{(i)}} x_{j} \\
= & \sum_{j=1}^{N}\left(\frac{1}{\left|V_{i}\right|} \sum_{u \in V_{i}} \frac{R_{u j}^{(i)}}{\sum_{k=1}^{N} R_{u k}^{(i)}}\right) x_{j} \\
= & \sum_{j=1}^{N} A_{i j} x_{j}
\end{aligned}
$$

where $A_{i j}$ is used to denote the inner summation term. The matrix $\mathbf{A}$ with elements $A_{i j}$ can be interpreted as a Retweet matrix that captures the tweet-and-retweet response feature in Twitter.

4. In our analysis of the 2012 U.S. presidential election, it is the Republican and Democratic Parties competing, and we assume liberals to support the Democrats/Obama, and conservatives to support the Republicans/Romney.

5. The specific forms of Eqs. (1) and (2) imply a user's contribution is limited in $[-1,1]$ regardless of the number of tweets/retweets it sends. If we treat all tweets the same, i.e., defining $y_{i}=$ $\sum_{u \in U_{i}} \sum_{t \in T_{u i}} s_{t} / \sum_{u \in U_{i}}\left|T_{i u}\right|$, the performance degrades probably due to the skew from a few highly vocal users.

6. We do not constrain $x_{j}$ to be bounded in $[-1,1]$, although $x_{j}$ and $y_{i}$ should be on the same scale, and a properly designed algorithm should be able to recover it.

7. In practice, we further restrict $U_{i}$ and $V_{i}$ to be the same user population by setting $U_{i}, V_{i} \leftarrow U_{i} \cap V_{i}$.

\subsection{An III-posed Linear Inverse Problem}

The main premise of this paper is that the behavior of tweeting and retweeting is consistent. Mathematically, we require the average tweet and retweet leanings per event to be similar:

$$
y_{i} \approx \sum_{j=1}^{N} A_{i j} x_{j}, \quad i=1, \ldots, E .
$$

Our goal is to choose $x_{j}$ 's that minimize the error from the consistency equations Eq. (4), where the error measure is chosen to be the sum of squared differences $\sum_{i}\left(\sum_{j} A_{i j} x_{j}-\right.$ $\left.y_{i}\right)^{2}$. Writing in matrix form, we are solving the unconstrained least squares problem

$$
\underset{\mathbf{x} \in \mathbb{R}^{N}}{\operatorname{minimize}} \quad\|\mathbf{A} \mathbf{x}-\mathbf{y}\|_{2}^{2} .
$$

We often have many more tweeters than events $(N=$ 1000, $E=12$ in Sections 5 and 6), then $N>E$ and the system of linear equations $\mathbf{A x}=\mathbf{y}$ is underdetermined, which means there are infinitely many solutions $\mathbf{x}$ that can achieve the minimum possible error of 0 in Problem (5). Then the problem becomes an ill-posed linear inverse problem [7]. The challenge of solving ill-posed problems is in selecting a reasonable solution out of the infinite set of feasible solutions. For example, in our initial studies, the least-norm solution to (5) yielded unsatisfactory results.

\subsection{Regularization}

In statistical inference, solving ill-posed problems requires us to incorporate prior knowledge of the problem to rule out undesirable solutions. One such common approach is regularization, and we can change the objective function in Problem (5), $\|\mathbf{A x}-\mathbf{y}\|_{2}^{2}$, to $\|\mathbf{A} \mathbf{x}-\mathbf{y}\|_{2}^{2}+\lambda f(\mathbf{x})$, where $\lambda>0$ is a regularization parameter, and $f(\mathbf{x})$ quantifies the "fitness" of a solution such that undesirable solutions have higher $f(\mathbf{x})$ values. For example, Tikhonov regularization for least-squares uses $f(\mathbf{x})=\|\mathbf{x}\|_{2}^{2}$ [7]. In this paper, we propose a regularization term that favors political leaning assignments $\mathbf{x}$ with $x_{i}$ being close to $x_{j}$ if sources $i$ and $j$ have similar retweet responses.

Let $W_{i j}$ be a regularization weight between sources $i$ and $j$ such that $W_{i j} \geq 0$ and $W_{i j}=W_{j i}$. Furthermore, let $\mathbf{W}$ be the weight matrix whose elements are $W_{i j}$. Then we set

$$
f(\mathbf{x})=\sum_{i=1}^{N} \sum_{j=1}^{N} W_{i j}\left(x_{i}-x_{j}\right)^{2},
$$

so that if $W_{i j}$ is large (sources $i$ and $j$ are similar), then $x_{i}$ should be close to $x_{j}$ to minimize $W_{i j}\left(x_{i}-x_{j}\right)^{2}$.

Note that $f(\mathbf{x})$ can be rewritten in terms of a graph Laplacian. Let $\mathbf{D}=\left[D_{i j}\right]$ be defined as

$$
D_{i j}= \begin{cases}\sum_{k=1}^{N} W_{i k} & i=j, \\ 0 & \text { otherwise }\end{cases}
$$

and $\mathbf{L}$ be the graph Laplacian defined as $\mathbf{L}=\mathbf{D}-\mathbf{W}$. Then it can be shown that

$$
\sum_{i=1}^{N} \sum_{j=1}^{N} W_{i j}\left(x_{i}-x_{j}\right)^{2}=2 \mathbf{x}^{T} \mathbf{L} \mathbf{x} .
$$




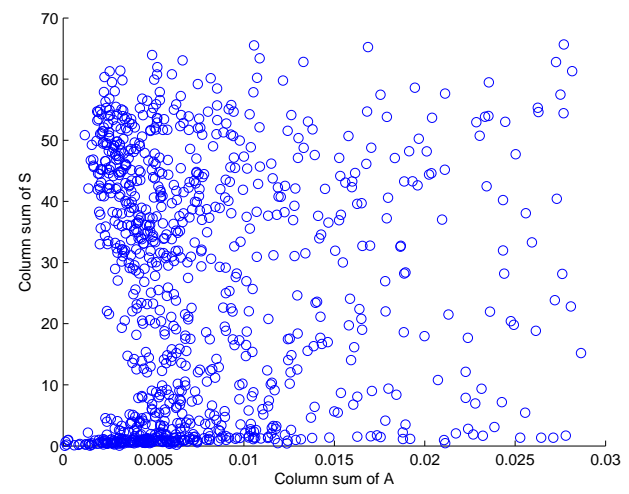

Fig. 4. Relationship between retweet popularity (column sum on $\mathbf{A}$ ) and regularization strength (column sum on $\mathbf{S}$ ) of top 1,000 retweet sources in Section 4. A large number of sources are on the bottom left corner, meaning they are both unpopular and insufficiently regularized.

Our $\mathbf{W}$ is constructed to account for the following.

Similarity based on co-retweeter sets. The first step in constructing $\mathbf{W}$ is to construct a similarity matrix $\mathbf{S}=\left[S_{i j}\right]$ that captures the similarity between two sources $i$ and $j$ by who retweeted them. Let $\mathcal{U}_{i}$ and $\mathcal{U}_{j}$ be the sets of users who have retweeted sources $i$ and $j$ respectively. We consider two standard similarity measures:

- Cosine similarity: $S_{i j}=\frac{\left|\mathcal{U}_{i} \cap \mathcal{U}_{j}\right|}{\sqrt{\left|\mathcal{U}_{i}\right| \cdot\left|\mathcal{U}_{j}\right|}}$, and

- Jaccard coefficient: $S_{i j}=\frac{\left|\mathcal{U}_{i} \cap \mathcal{U}_{j}\right|}{\left|\mathcal{U}_{i} \cup \mathcal{U}_{j}\right|}$.

Individualizing regularization strength. Regularization is more important for sources with insufficient information available from A: if source $i$ does not get retweeted often, her corresponding column sum, $\sum_{j} A_{j i}$, is small, and inferring her score $x_{i}$ based on the error term $\|\mathbf{A x}-\mathbf{y}\|_{2}^{2}$ suffers from numerical stability issues.

In practice, a source can simultaneously be scarcely retweeted and have low similarity with other sources, i.e., source $i$ has $S_{i j}$ small for all other sources $j$. If $\mathbf{S}$ is directly used for regularization (i.e., by setting $\mathbf{W} \leftarrow \mathbf{S}$ ), the source is insufficiently regularized and inference becomes unreliable. See Figure 4 for confirmation from real data.

Our solution to this problem is to apply matrix scaling [35] to $\mathbf{S}$. We compute $\mathbf{W}$ as the matrix closest to $\mathbf{S}$ (under an Kullback-Leibler divergence-like error function) such that the row and column sums of $\mathbf{W}$ satisfy equality constraints:

$$
\begin{array}{llr}
\underset{\mathbf{W} \geq 0}{\operatorname{minimize}} & \sum_{i, j=1}^{N} W_{i j} \log \frac{W_{i j}}{S_{i j}} & \\
\text { subject to } & W_{i j}=0 & \text { for }(i, j) \text { s.t. } S_{i j}=0 \\
& \sum_{j=1}^{N} W_{i j}=u_{i} & i=1, \ldots, N \\
& \sum_{j=1}^{N} W_{j i}=u_{i} & i=1, \ldots, N,
\end{array}
$$

where $\mathbf{u}=\left\{u_{i}\right\}$ is a "regularization strength" parameter vector.

Problem (8) is solved by iterated rescaling of the rows and columns of $\mathbf{S}$ until convegence [35]. If the resultant $\mathbf{W}$

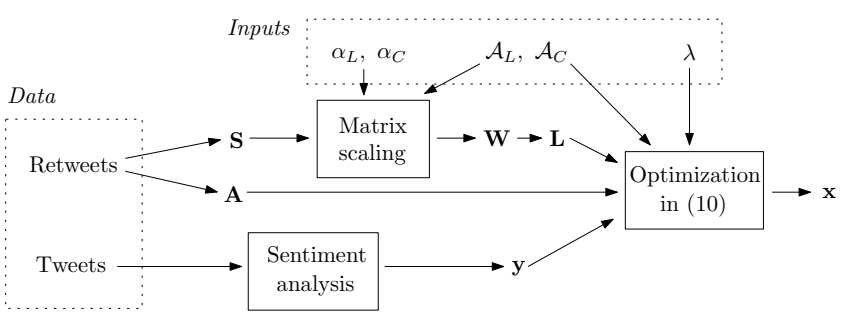

Fig. 5. Flowchart of data processing and inference steps.

is asymmetric, we take the transformation $\mathbf{W} \leftarrow$ ( $\mathbf{W}+$ $\left.\mathbf{W}^{T}\right) / 2$. It can be shown that the transformed $\mathbf{W}$ also satisfies the constraints in (8).

Incorporating prior knowledge. Prior knowledge can readily be incorporated into our inference technique as constraints of an optimization problem. In this paper we consider two types of prior knowledge:

- Anchors: sources carrying an extreme leaning, e.g., the election candidates themselves, can serve as anchors with fixed political leaning $x_{i}$. In the literature this idea has been used frequently [21, 3, 19].

- Score distribution: $u_{i}$ can be interpreted as the strength of influence that source $i$ exerts on sources similar to herself. Intuitively, anchors should exert higher influence, because we are more confident in their political leaning. Therefore, we set $\mathbf{u}$ as:

$$
u_{i}= \begin{cases}\alpha_{L} & i \in \mathcal{A}_{L} \\ \alpha_{C} & i \in \mathcal{A}_{C} \\ 1 & \text { otherwise }\end{cases}
$$

where $\alpha_{L}$ and $\alpha_{C}$ are tuning parameters, and $\mathcal{A}_{L}$ and $\mathcal{A}_{C}$ are the sets of liberal and conservative anchors respectively. Given there should exist some non-anchor sources with extreme leaning, we tune $\alpha_{L}$ and $\alpha_{C}$ as follows: (a) initialize $\alpha_{L}=\alpha_{C}=1$, and then (b) iteratively increase $\alpha_{L}$ and $\alpha_{C}$ until the computed most extreme political leaning of a nonanchor source is within $90 \%$ of that of an anchor.

\subsection{Optimization Problem}

Combining Eqs. (5), (6) and knowledge of anchors, ${ }^{8}$ our final optimization formulation is:

$$
\begin{array}{cll}
\underset{\mathbf{x} \in \mathbb{R}^{N}}{\operatorname{minimize}} & \|\mathbf{A} \mathbf{x}-\mathbf{y}\|_{2}^{2}+\lambda \mathbf{x}^{T} \mathbf{L x} & \\
\text { subject to } & x_{i}=-1 & i \in \mathcal{A}_{L} \\
& x_{i}=1 & i \in \mathcal{A}_{C},
\end{array}
$$

where $\lambda$ is a tuning parameter. Figure 5 summarizes the data processing and inference steps in Section 3.

\subsection{Extension: Sparsifying graph Laplacian.}

Since most of the sources we consider are popular, most pairs of sources have at least one user who has retweeted both of them, and $\mathbf{S}$ is likely to be dense (in our dataset, $83 \%$

8. We define a liberal (conservative) anchor to have a negative (positive) score to be consistent with the convention that liberals (conservatives) are on the "left" ("right"). 
of its entries are nonzero). From a computational standpoint it is advantageous to sparsify the matrix, so we also evaluate our algorithm with an extra $k$-nearest-neighbor step, such that $S_{i j}$ is kept only if $j$ is a nearest neighbor of $i$, or vice versa. We are able to obtain good performance even when $\mathbf{S}$ is less than $10 \%$ sparse. See Section 6.4 for details.

\section{Dataset}

In this section we describe the collection and processing of our Twitter dataset of the U.S. presidential election of 2012. Our dataset was collected over a timespan of seven months, covering from the initial phases to the climax of the campaign.

Data Collection. From April 8 to November 10 2012, we used the Twitter streaming $\mathrm{API}^{9}$ to collect 119 million tweets which contain any one of the following keyword phrases: "obama", "romney", "barack", "mitt", "paul ryan", "joe biden", "presidential", "gop", "dems", "republican" and "democrat" (string matching is case-insensitive).

Event Identification. By inspecting the time series of tweet counts in Figure 6, we manually identified 12 events as listed in Table 1. We defined the dates of an event as follows: the start date was identified based on our knowledge of the event, e.g., the start time of a presidential debate, and the end date was defined as the day when the number of tweets reached a local minimum or dropped below that of the start date. After the events were identified, we extracted all tweets in the specified time interval ${ }^{10}$ without additional filtering, assuming all tweets are relevant to the event and those outside are irrelevant.

Extracting Tweet Sentiment. We applied SentiStrength [36], a lexicon-based sentiment analysis package, to extract the sentiment of tweets. We adjusted the provided lexicon by compiling a high-frequency tweet-word list per event, and then removing words ${ }^{12}$ that we consider to not carry sentiment in the context of elections. Sentiment analysis was done as a ternary (positive, negative, neutral) classification.

For each tweet $t$, we set its score $s_{t}=-1$ if either (a) it mentions solely the Democrat camp (has "obama", "biden" etc. in text) and is classified to have positive sentiment, or (b) it mentions solely the Republican camp ("romney", "ryan" etc.) and has negative sentiment. We set $s_{t}=1$ if the opposite criterion is satisfied. If both criteria are not satisfied, we set $s_{t}=0$.

\section{Evaluation}

\subsection{Ground truth construction}

We compare the political leaning scores learnt by our technique with "ground truth" constructed by human evaluation. First, 100 sources are randomly selected from the 1,000 most popular retweet sources. ${ }^{13}$ Then we ask 12 human

9. https://dev.twitter.com/streaming/overview

10. For retweets, we only include those with the original tweet being created within the time interval.

11. A time interval starts at 00:00:00 of start date, and ends at 23:59:59 of end date. Timezone used is UTC.

12. They are "gay" (as in "gay marriage"), "foreign" ("foreign policy"), "repeal" ("repeal obamacare") and "battle" ("battleship").

13. Those who were retweeted the largest cumulative number of times during the 12 events. judges with sufficient knowledge of American politics to classify each of the 100 sources as "L" (Liberal, if she is expected to vote Obama), "C" (Conservative, if expected to vote Romney), or " $\mathrm{N}$ " (Neutral, if she cannot decide), supposing each source is one voter who would vote in the presidential election. For each source, a judge is presented with (a) the source's user profile, including screen name, full name, self description, location and etc., and (b) ten random tweets published by the source. Given the set of labels, we compute our ground truth political leaning scores $\left\{\tilde{x}_{i}\right\}$ as follows: for each label of L/N/C, assign a score of $-1 / 0 /+1$, then the score of source $i$, call it $\tilde{x}_{i}$, as the average of her labels.

While there are many alternatives to defining and constructing ground truth, our choice is motivated by our implicit assumption of Twitter political leaning being the perceived leaning by a source's retweeters. If source $i$ has $\tilde{x}_{i}$ with extreme values $(-1$ or +1$)$, then it is unambiguously liberal/conservative, but if $\tilde{x}_{i}$ takes intermediate values, then some human judges may be confused with the source's leaning, and the general Twitter population is likely to have similar confusion, which suggests that the "correct" $x_{i}$ should also take intermediate values. Defining $\left\{\tilde{x}_{i}\right\}$ this way also allows us to understand the usefulness of quantifying political leaning with a continuous score. Obviously, if all $\tilde{x}_{i}$ are either -1 or +1 , a simple binary classification of the sources is enough, but as we see in Figure $7,\left\{\tilde{x}_{i}\right\}$ is evenly spread across the range of allowed values $[-1,1]$, so characterizing sources with simple binary, or even ternary, classification appears too coarse. To further support our claim, we also compute the inter-rater agreement of our manual labels as Fleiss' $\kappa=0.430$ [14], a moderate level of agreement [23]. This suggests that while the labels are reliable, classifying sources is not trivial and a continuous political leaning score is useful.

Finally, we also manually classify each of the 1,000 most popular sources into four classes:

- Parody: role-playing and joke accounts created for entertainment purposes (example joke tweet: "I cooked Romney noodles Obama self," a pun on "I cooked ramen noodles all by myself")

- Political: candidates of the current election and accounts of political organizations

- Media: outlets for distributing information in an objective manner, setting aside media bias issues

- Others: personal accounts, including those of celebrities, pundits, reporters, bloggers and politicians (excluding election candidates).

\subsection{Performance metrics}

The quality of political leaning scores is measured under two criteria.

Classification. One should be able to directly infer the liberal/conservative stance of a source $i$ from her sign of $x_{i}$, i.e., it is liberal if $x_{i}<0$, or conservative if $x_{i}>0$. Taking $\left\{\tilde{x}_{i}\right\}$ as ground truth, we say source $i$ is correctly classified if the signs of $x_{i}$ and $\tilde{x}_{i}$ agree. ${ }^{14}$ Classification performance is

14. In the unlikely case of $\tilde{x}_{i}=0$ ( 2 out of 100 test cases), we require $x_{i}=0$ for correct classification. 


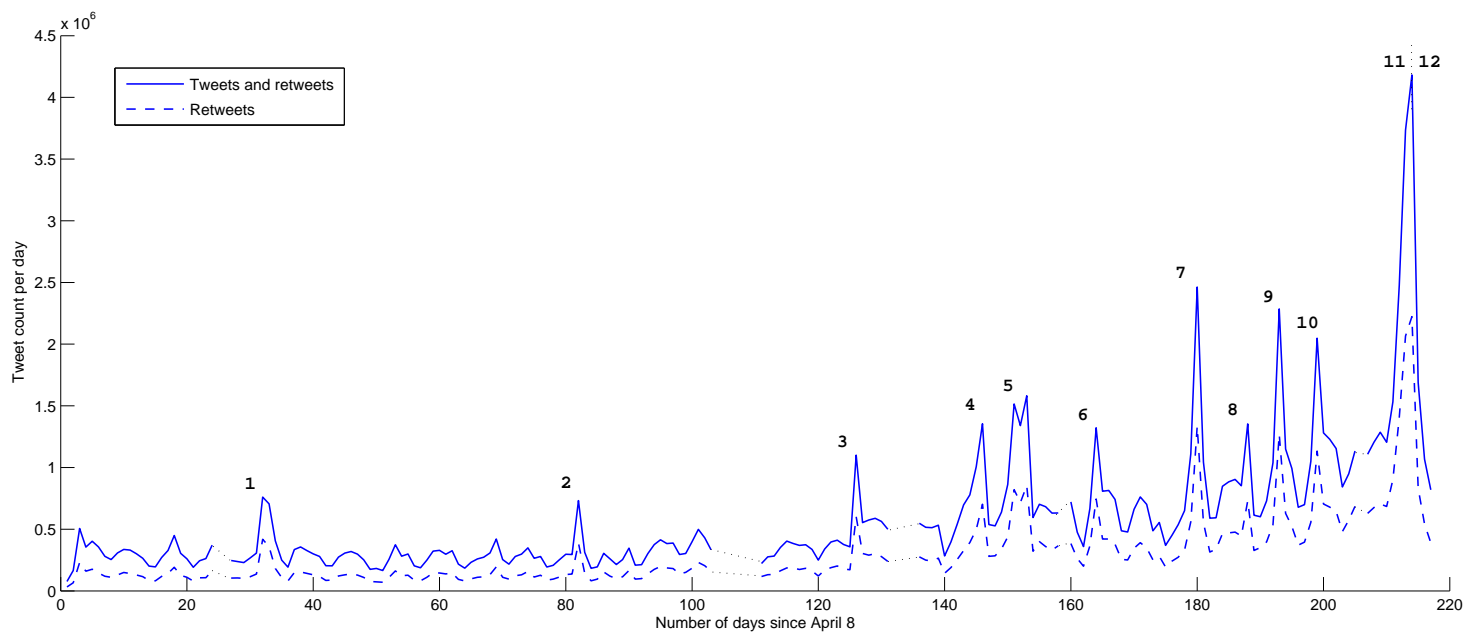

Fig. 6. Number of tweets per day. Numbers on plot indicate events (see Table 1), and dotted lines indicate time periods when significant data were lost due to network outage (five instances).

TABLE 1

Summary of events identified in the dataset.

\begin{tabular}{c|l|l|c|c}
\hline ID & Dates $^{11}$ & Description & \# tweets (m) & \# non-RT tweets (m) \\
\hline 1 & May 9 - 12 & Obama supports same-sex marriage & 2.10 & 1.35 \\
2 & Jun 28 - 30 & Supreme court upholds health care law & 1.21 & 0.78 \\
3 & Aug 11 - 12 & Paul Ryan selected as Republican VP candidate & 1.62 & 0.96 \\
4 & Aug 28 - Sep 1 & Republican National Convention & 4.32 & 2.80 \\
5 & Sep 4 - 8 & Democratic National Convention & 5.81 & 3.61 \\
6 & Sep 18 - 22 & Romney's 47 percent comment & 4.10 & 2.55 \\
7 & Oct 4 - 5 & First presidential debate & 3.49 & 2.19 \\
8 & Oct 12 - 13 & Vice presidential debate & 1.92 & 1.19 \\
9 & Oct 17 - 19 & Second presidential debate & 4.38 & 2.67 \\
10 & Oct 23 - 26 & Third presidential debate & 5.62 & 3.35 \\
11 & Nov 4 - 6 & Elections (before Obama projected to win) & 7.50 & 4.40 \\
12 & Nov 7 - 9 & Elections (after Obama projected to win) & 6.86 & 4.43 \\
\hline Total & & & 48.90 & 30.28 \\
\hline
\end{tabular}

measured using the standard metrics of accuracy, precision, recall and F1 score.

Rank correlation. The set of scores $\left\{x_{i}\right\}$ induce a ranking of the sources by their political leaning. This ranking should be close to that induced by the ground truth scores $\left\{\tilde{x}_{i}\right\}$. We measure this aspect of performance using Kendall's $\tau$, which varies from -1 (perfect disagreement) to 1 (perfect agreement).

\subsection{Results}

We solve Problem (10) with $\mathcal{A}_{L}=\{$ Obama2012 $\}$ and $\mathcal{A}_{C}=\{$ MittRomney $\}$ and compare the results with those from several baselines:

- PCA: we run Principal Components Analysis on A with each column being the feature vector of a source, with or without the columns being standardized, and take the first component as $\left\{x_{i}\right\}$. This is the baseline when we use only the information from $\mathbf{A}$ (retweet counts).

- Eigenvector: we compute the second largest eigenvector of $\mathbf{L}$, with $\mathbf{L}$ becoming computed from $\mathbf{S}$ being either the cosine or Jaccard matrix. This is a technique commonly seen in spectral graph partitioning [12], and is the standard approach when only the information from $\mathbf{S}$ (retweeters) is available. Note that the $\mathbf{x}$ computed this way is equivalent to solving the optimization problem: minimize $\mathbf{x}^{T} \mathbf{L} \mathbf{x}$, subject to $\|\mathbf{x}\|_{2}=1, \mathbf{x}^{T} \mathbf{1}=0$.

- Sentiment analysis: we take $x_{i}$ as the average sentiment of the tweets published by source $i$, using the same methdology in computing $\mathbf{y}$ [36]. This is the baseline when only tweets are used.

Table 2 reports the evaluation results. Our algorithm, in combining information from $\mathbf{A}, \mathbf{S}$ and $\mathbf{y}$, performs significantly better than all baselines in terms of Kendall's $\tau$, F1 score and accuracy. We also observe that if no matrix scaling is applied in constructing $\mathbf{W}$, the algorithm tends to assign all $\left\{x_{i}\right\}$ (except those of anchors) to the same sign, resulting in poor classification performance. Somewhat surprisingly, sentiment analysis performs the best among all the baselines, in contrast to what one would expect based on related work [28, 10,33]. In the remaining of this paper, we focus on the political leaning scores computed using cosine similarity.

Figure 7 shows the correspondence between $\left\{x_{i}\right\}$ and $\left\{\tilde{x}_{i}\right\}$. The two sets of scores exhibit similar rankings of sources and a bimodal score distribution. The scores due 
TABLE 2

Performance of our algorithm compared to several baselines. Best two results (almost always due to our method) are highlighted in bold.

\begin{tabular}{|c|c|c|c|c|c|c|c|c|}
\hline Algorithm & Kendall's $\tau$ & Precision, L & Recall, L & Precision, C & Recall, C & F1 score, L & F1 score, C & Accuracy \\
\hline Ours, cosine matrix & 0.652 & 0.942 & 0.970 & 0.935 & 0.935 & 0.955 & 0.935 & 0.94 \\
\hline Ours, Jaccard matrix & 0.654 & 0.940 & 0.940 & 0.879 & 0.935 & 0.940 & 0.906 & 0.92 \\
\hline Ours, cosine $\mathrm{w} / \mathrm{o}$ scaling & 0.649 & 0.670 & 1 & 0 & 0 & 0.802 & 0 & 0.67 \\
\hline Ours, Jaccard w/o scaling & 0.641 & 0 & 0 & 0.31 & 1 & 0 & 0.473 & 0.31 \\
\hline PCA & 0.002 & 0.663 & 0.791 & 0.300 & 0.194 & 0.721 & 0.235 & 0.59 \\
\hline PCA, standardized columns & 0.011 & 0.750 & 0.224 & 0.325 & 0.839 & 0.345 & 0.468 & 0.41 \\
\hline Eigenvector, cosine matrix & 0.370 & 0.838 & 0.851 & 0.688 & 0.710 & 0.844 & 0.698 & 0.79 \\
\hline Eigenvector, Jaccard matrix & 0.292 & 0.864 & 0.761 & 0.610 & 0.806 & 0.810 & 0.694 & 0.76 \\
\hline Sentiment analysis & 0.511 & 0.926 & 0.746 & 0.700 & 0.903 & 0.826 & 0.789 & 0.78 \\
\hline
\end{tabular}
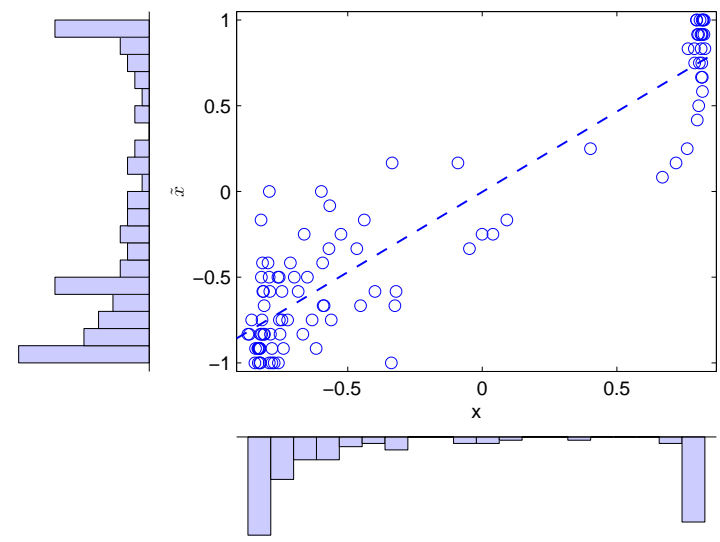

Fig. 7. Relationship of ground truth $\left\{\tilde{x}_{i}\right\}$ and our computed scores $\left\{x_{i}\right\}$ on the 100 sources with manual labels, together with their marginal distributions. Our method is able to recover both the correct classifications (datapoints in bottom-left and upper-right quadrants) and rankings for most sources.

to our algorithm are slightly more polarized.

\section{Numerical Study}

\subsection{Quantifying Prominent Retweet Sources}

We study the properties of the political leaning of the 1,000 most popular retweet sources. Similar to that in Figure 7, the score histogram on the full set (Figure 8) has a bimodal distribution. We note that by incorporating retweeter information, our algorithm is able to correctly position "difficult" sources that were highly retweeted during events unfavorable to the candidate they support, e.g., JoeBiden, CBSNews and all accounts related to Big Bird, an improvement over the preliminary version of this paper [42]. We also find that WSJ is assigned a slightly liberal score. This is consistent with findings reported in prior studies [20,26] explained by the separation between WSJ's news and editorial sections.

We also study the score distributions of sources grouped by account type. Figure 9 shows noticeable differences among the different groups. Parody sources are skewed towards the liberal side. Political sources are strongly polarized with no sources having neutral (close to zero) scores. Media sources are less polarized with a more even spread of scores. Sources in the "Others" class have a score distribution close to that of political sources, but also includes a few neutral scores, which can be attributed to celebrities with no clear political stance.
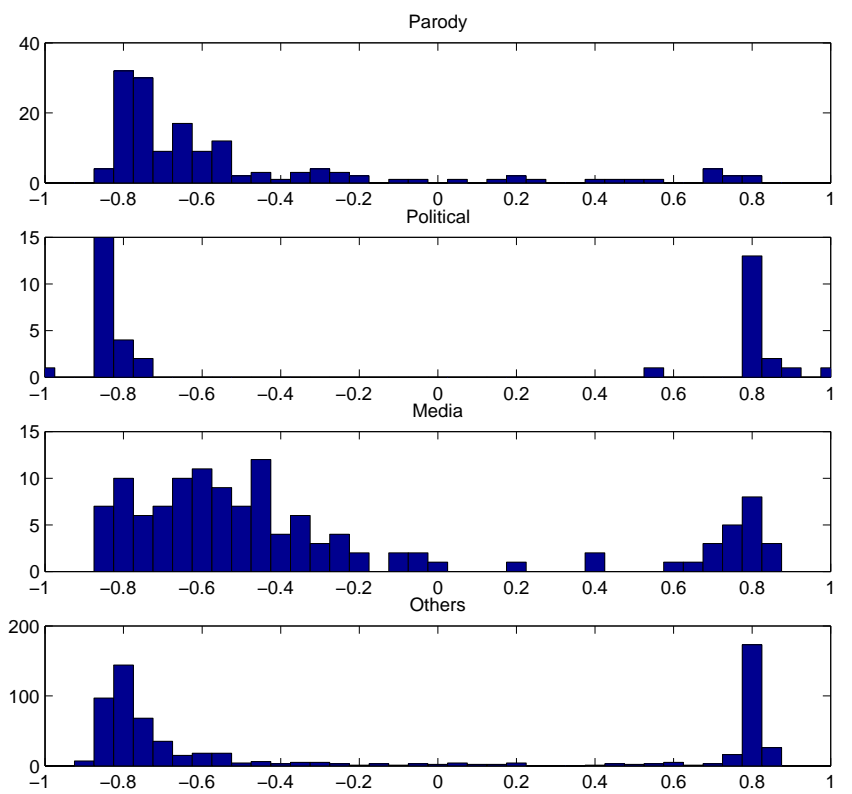

Fig. 9. Distribution of political leaning scores grouped by Twitter account type. Parody/comedy accounts are skewed towards the liberal side. Political accounts are more polarized (no scores close to zero) than other accounts.

\subsection{Quantifying Ordinary Twitter Users}

Given the political leaning of 1,000 retweet sources, we can use them to infer the political leaning of ordinary Twitter users who have retweeted the sources. We consider the set of users seen in our dataset who have retweeted the sources at least ten times, including retweets made during nonevent time periods. In total there are 232,000 such users. We caution this set of users is not necessarily representative of the general Twitter population, or even the full population of our dataset (9.92 million users in total), but we believe it is possible to "propagate" score estimates from these 232,000 users to everyone else, which remains as future work.

For user $u$, we infer her political leaning $x_{u}$ as

$$
x_{u}=\frac{\sum_{i=1}^{N} R_{u i} x_{i}}{\sum_{i=1}^{N} R_{u i}},
$$

where $R_{u i}$ is the number of times user $u$ retweeted source $i$ and $x_{i}$ is source $i$ 's political leaning.

Figure 10 shows the kernel density estimate of the political leaning scores of the 232,000 ordinary users, compared with that of the set of 1,000 sources. These users have a slightly less polarized distribution (density function is closer 


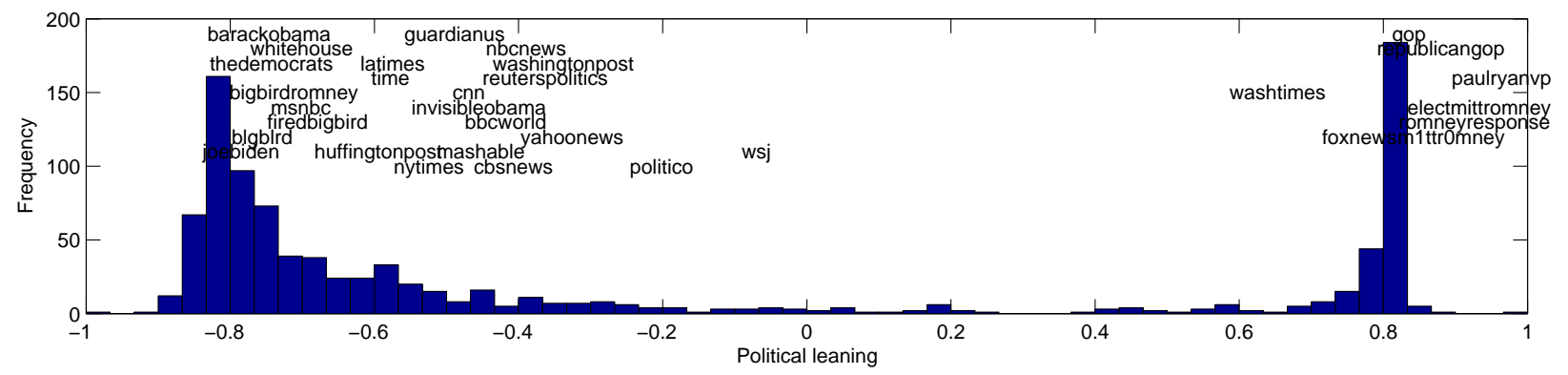

Fig. 8. Distribution of political leaning scores of top 1,000 retweet sources with positions of some example sources.

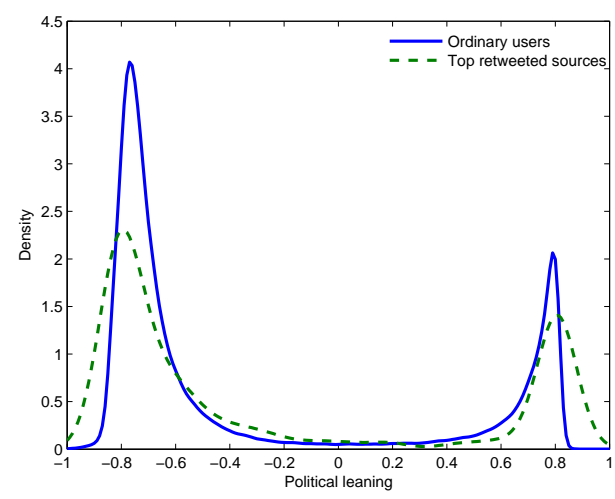

Fig. 10. Kernel density estimates of political leaning of top 1,000 retweet sources and 232,000 ordinary Twitter users.

to zero), but are more skewed towards the liberal side ( $72.5 \%$ have $x_{u}<0$, compared to $69.5 \%$ for retweet sources).

Measuring polarization. Using the learnt political leaning scores, we aim to quantify political polarization of a population, but for this to be possible, we first need a polarization measure. Let us consider one user $u$. A natural measure $P_{u}$ for her polarization can be defined as how far her political leaning is away from neutral: $P_{u}=\left|x_{u}-0\right|=\left|x_{u}\right|$. Then for a population $\mathcal{U}$, its polarization measure can be taken as the average of all $P_{u}$ for $u \in \mathcal{U}$. However, such a definition does not account for class imbalance (liberals outnumbering conservatives in our case), so we take a classbalanced definition instead:

$$
P_{\mathcal{U}}=\frac{1}{\left|\mathcal{U}_{+}\right|} \sum_{u \in \mathcal{U}_{+}} x_{u}+\frac{1}{\left|\mathcal{U}_{-}\right|} \sum_{u \in \mathcal{U}_{-}}\left|x_{u}\right|
$$

where $\mathcal{U}_{+}=\left\{u \mid u \in \mathcal{U}, x_{u}>0\right\}$ and $\mathcal{U}_{-}=\{u \mid u \in$ $\left.\mathcal{U}, x_{u}<0\right\}$.

Now we put the 232,000 ordinary users into ten percentile bins, such that the first bin contains the lowest $10 \%$ of users according to their retweet activity (number of retweets made, including retweets of non-top 1,000 sources), then the next bin contains the next lowest $10 \%$, and so on. Figure 11 shows the plot of two skew measures: the polarization measure as defined in Eq. (12), and the fraction of liberals $\left|\mathcal{U}_{+}\right| /\left(\left|\mathcal{U}_{+}+\mathcal{U}_{-}\right|\right)$. We observe that liberals dominate (>80\%) the population of low activity users, but as retweet activity increases, the liberal-conservative split becomes more balanced (roughly $50 \%$ in the last bin). This suggests that most Twitter users $(80 \%$ of them make less than 100 retweets

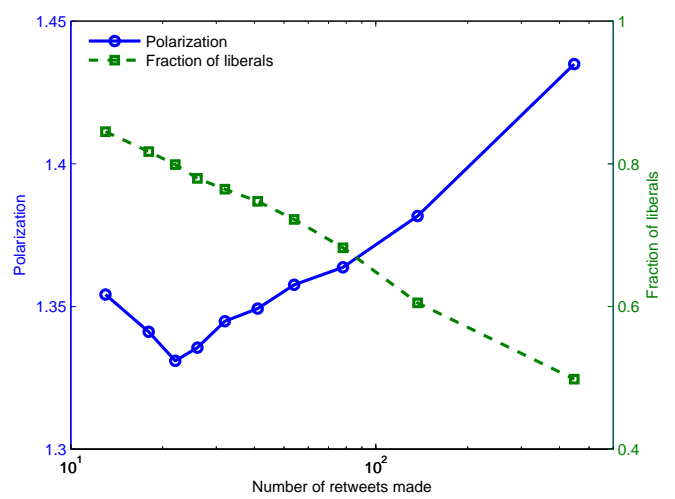

Fig. 11. Skew measures of ordinary users binned by $10 \%$-tiles of rewteet activity. Polarization and liberal-conservative balance increase for more active populations.

on the election) tend to be liberals, but the most vocal population (those making 100 to 33,000 retweets) consists of users who are more politically opiniated such that they spend more effort to promote their causes in social media. This is supported by the plot of the polarization measure, which increases with retweet activity.

\subsection{Temporal Dynamics}

With the large amount of data available from Twitter one can perform fine-grained temporal analysis using data from a relatively short timespan. In this section, we study how many events are necessary to obtain sufficiently good performance, and then present two examples in applying our methodology to social media monitoring.

Stability. Here we quantify the political leaning of the 1,000 sources studied in Section 6.1 but with varying amounts of information. We start by running our inference technique using data from only the first event, then we use events 1 to 2 , and so on. Then each source has a sequence of 12 political leaning scores, and we evaluate the quality of these scores compared to ground truth. Figure 12(a) shows that three events, or $10 \%$ of the data, are enough for achieving performance close to that from using the full dataset. From the description of the events in Table 1, the first two events are skewed towards the liberal side, and it is not clear how conservative users react to them (either object strongly or remain silent). With the third event added, we have a more balanced set of data for reliable inference. 
Next we look at the stability of scores per source across the 12 events. Figure 12(b) plots the mean deviation of a source's score per event from her final score. Using the classification from Section 5, we see stability varies with the type of a source. Political sources are the most stable with their score deviations having decreased quickly after three events, consistent with our intuition that they are the easy cases with the least ambiguity in political leaning. On the other hand, parody accounts are the least stable. Their patterns of being retweeted are the least stable with large fluctuations in retweet counts across different events, resulting in less reliable inference. Also, users do not retweet parody sources by how agreeable, but rather by how funny they are, so even retweeter information on these sources is less stable across different events.

Trending hashtags. The political leaning of hashtags [41] can be quantified by how they are being used by Twitter users. Here we consider a simple way to estimate it using the political leaning scores of the top retweet sources. For each hashtag, we compute its political leaning as the average of all sources (within the top 1000 retweet sources) that have used it at least once in their published tweets. Moreover, we perform this computation per event (excluding previous events) to obtain a sequence of hashtag political leaning scores. This allows us to track trending hashtags as events unfold.

Tables 3 and 4 are the lists of the most liberal or conservative hashtags that have been used by at least ten sources in each of the events. Besides the static hashtags indicating users' political affiliation such as \#TCOT (top conservatives on twitter) and \#p2b (progressive 2.0 bloggers), we discover hashtags being created in response to events (\#ssm (samesex marriage) in event 1, \#MyFirstTime in event 10 for a suggestive Obama ad). Moreover, there are hashtags showing opposite opinions on the same issue (\#aca (affordable care act) vs \#ObamaTax in event 2, \#WrongAgainRyan vs \#BidenUnhinged in event 8), and many instances of sarcasm (\#StopIt and \#YouPeople in event 6, \#notoptimal in event 9 for Obama saying the government's response was "not optimal" during the Benghazi attack) and accusations (\#MSM (main stream media) for liberal media bias).

In the latter half of the dataset, liberals tend to focus on accusing the other side of lying during the debate (\#LyinRyan, \#SketchyDeal, \#MittLies, \#AdmitItMitt), while conservatives tend to focus on the Benghazi attack (\#BenghaziGate, \#notoptimal, \#7HoursOfHell, \#Benghazi). Finally, we note a change in hashtag usage before and after the election outcome came out (from \#WhyImNotVotingForRomney to \#FourMoreYears).

Tracking temporal variation in polarization. We begin with this question: is the Twitter population more (or less) polarized during an event?

Without analyzing the data, the answer is not clear because it is influenced by two factors with opposite effects:

- An event draws attention from less vocal users who are likely to have weak political leaning. These users join the discussion because everyone talks about it.

- On the other hand, the fact that a usually silent user joining the discussion may indicate he/she is strongly opinionated about the topic of discussion.
To answer the question, we compute the polarization measure in Eq. (12) for each day in our dataset, with population $\mathcal{U}$ taken as the set of users (out of the 232,000 users from Section 6.2) who have tweeted or retweeted on that day. For comparison purposes we also compute the fraction of liberals per day.

The results are shown in Figure 13. First, liberals outnumber conservatives in every single day, regardless of whether it is an event day or not. Second, there is a slight increasing trend in polarization over the course of events, which corresponds to discussions become more heated as the election campaign progresses. Third, the fraction of liberals is significantly higher at the onset (first day) of an event. This is observed in 10 out of the 12 events. In contrast, polarization drops and reaches a local minimum during an event (10/12 events), and the level is lower than nearby nonevent days. It appears the influx of users during an event drives polarization of the Twitter population down, because these extra users tend to have weaker political leaning.

We also contrast the changes in liberal skew and polarization right before and after the election outcome came out: at the climax of the election, the liberal-conservative share in active users is relatively balanced because both sides want to promote their candidate of support; at the same time the population is more polarized. After Obama is projected to win, there is a jump in liberal fraction with conservatives leaving the discussion, and polarization plummets, probably with the departure of strong-leaning conservatives.

\subsection{Sensitivity to Parameter Variation}

Our algorithm is robust to variation in input parameters $\lambda$, $\alpha_{C}$ and $\alpha_{L}$. Starting from the chosen $\left(\lambda, \alpha_{C}, \alpha_{L}\right)$ tuple from the previous section, we fix two parameters and vary the remaining one. Figures 14(a) and 14(c) show the resultant performance does not vary significantly over a wide range of parameter values.

We also consider a simple approach to sparsify the graph Laplacian. Given S, we preprocess it with a $k$-nearestneighbor step before passing it to matrix scaling: for each source $i$ we find the $k$ other sources $\{j\}$ with highest $S_{i j}$, then we keep only the $S_{i j}$ entries (not set to zero) for $j$ being a neighbor of $i$ or $i$ being a neighbor of $j$. We vary the value of $k$ from 50 to 1000 to vary the sparsity of $\mathbf{S}$ (and $\mathbf{L}$ ). Figure 14(b) shows even when $k$ is small $(k=50$ results in a sparsity of $8.7 \%$ ), the performance is still very good. ${ }^{15}$

\section{CONCLUSIONS AND FUtURE WORK}

Scoring individuals by their political leaning is a fundamental research question in computational political science. From roll calls to newspapers, and then to blogs and microblogs, researchers have been exploring ways to use bigger and bigger data for political leaning inference. But new challenges arise in how one can exploit the structure of the data, especially because bigger data are often are noisier and sparser.

In this paper, we propose to leverage two properties of Twitter data: (a) Twitter users tend to tweet and

15. Reducing $k$ further introduces convergence problems in the matrix scaling step. 


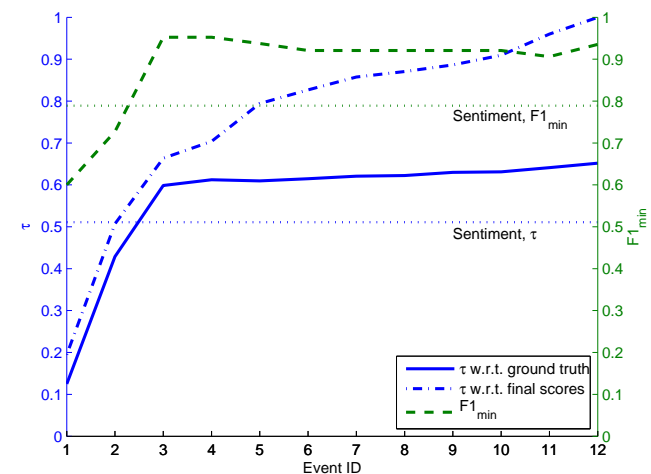

(a) Performance measures. Denote $\mathrm{F} 1_{\min }$ as the minimum of F1 scores of $\mathrm{C}$ and L classes.

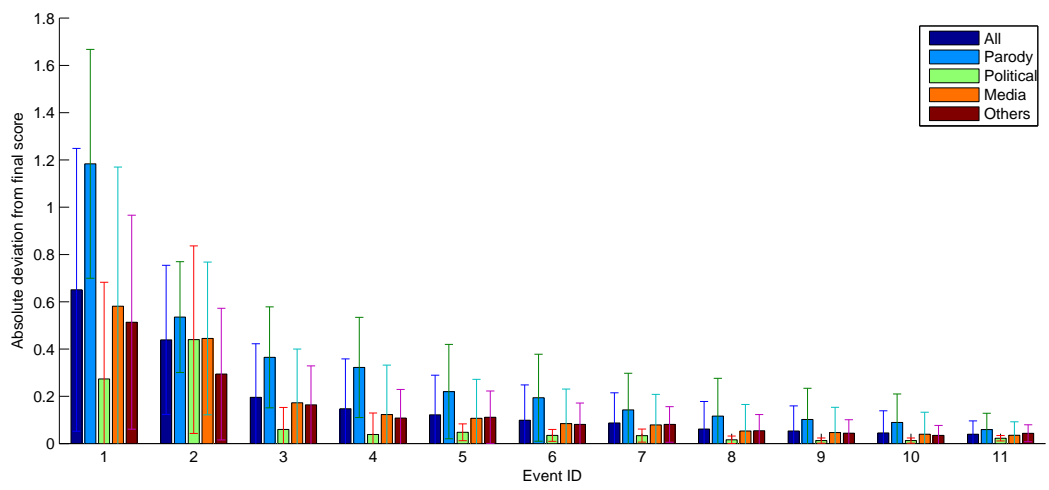

(b) Deviation from final result, with grouping by account type.

Fig. 12. Stability of results with varying number of events: good performance is achieved with only the first three events, and parody accounts are the least stable.

TABLE 3

Hashtags with highest liberal political leaning per event.

\begin{tabular}{|c|c|c|c|c|c|}
\hline Event & \multicolumn{5}{|c|}{ Top five liberal hashtags } \\
\hline 1 & \#MarriageEquality & \#equality & \#marriageequality & \#edshow & \#ssm \\
\hline 2 & \#aca & $\# p 2 b$ & \#ACA & \#Obama2012 & \#edshow \\
\hline 3 & $\# \mathrm{p} 2 \mathrm{~b}$ & \#Medicare & \#topprog & \#edshow & \#Obama2012 \\
\hline 4 & \#p2b & \#YouPeople & \#Current2012 & \#msnbc2012 & \#uppers \\
\hline 5 & \#ACA & \#PaulRyan & \#LyinRyan & \#nerdland & \#DavidGregorysToughQuestions \\
\hline 6 & \#LyinRyan & \#ObamaBiden2012 & \#StopIt & $\# \mathrm{p} 2 \mathrm{~b}$ & \#YouPeople \\
\hline 7 & \#47Percent & \#47percent & \#Forward & \#TeamObama & \#topprog \\
\hline 8 & \#WrongAgainRyan & $\# p 2 b$ & \#Bain & \#Sensata & \#LyinRyan \\
\hline 9 & \#SketchyDeal & \#Bain & \#MittLies & $\# \mathrm{p} 2 \mathrm{~b}$ & \#BinderFullofWomen \\
\hline 10 & \#StrongerWithObama & \#RomneyWrong & \#ObamaBiden2012 & \#RomneyNotReady & \#AdmitItMitt \\
\hline 11 & $\# p 2 b$ & \#uppers & \#msnbc2012 & \#ObamaBiden2012 & \#WhyImNotVotingForRomney \\
\hline 12 & \#OBAMA & \#obama2012 & \#msnbc2012 & \#Boehner & \#FourMoreYears \\
\hline
\end{tabular}

TABLE 4

Hashtags with highest conservative political leaning per event.

\begin{tabular}{|c|c|c|c|c|c|}
\hline Event & \multicolumn{5}{|c|}{ Top five conservative hashtags } \\
\hline 1 & \#LNYHBT & \#lnyhbt & \#twisters & \#sgp & \#ocra \\
\hline 2 & \#LNYHBT & \#ObamaTax & \#Obamatax & \#ocra & \#lnyhbt \\
\hline 3 & \#LNYHBT & \#sgp & \#TCOT & \#Mitt2012 & \#ocra \\
\hline 4 & \#LNYHBT & \#twisters & \#ocra & \#sgp & \#TCOT \\
\hline 5 & \#Mitt2012 & \#LNYHBT & \#ocra & \#twisters & \#military \\
\hline 6 & \#ObamaIsntWorking & \#WAR & \#Resist44 & \#2016 & \#MSM \\
\hline 7 & \#EmptyChair & \#ForwardNotBarack & \#sgp & \#resist44 & \#TCOT \\
\hline 8 & \#BenghaziGate & \#sgp & \#CNBC2012 & \#BidenUnhinged & \#lnyhbt \\
\hline 9 & \#CantAfford4more & \#BenghaziGate & \#notoptimal & \#sgp & \#Missouri \\
\hline 10 & \#BenghaziGate & \#Benghazigate & \#sgp & \#caring & \#MyFirstTime \\
\hline 11 & \#military & \#7HoursOfHell & \#Twibbon & \#LNYHBT & \#BENGHAZI \\
\hline 12 & \#sgp & \#ocra & \#Benghazi & \#WAR & \#lnyhbt \\
\hline
\end{tabular}

retweet consistently, and (b) similar Twitter users tend to be retweeted by similar sets of audience, to develop a convex optimization-based political leaning inference technique that is simple, efficient and intuitive. Our method is evaluated on a large dataset of 119 million U.S. election-related tweets collected over seven months, and using manually constructed ground truth labels, we found it to outperform many baseline algorithms. With its reliability validated, we applied it to quantify a set of prominent retweet sources, and then propagated their political leaning to a larger set of ordinary Twitter users and hashtags. The temporal dynamics of political leaning and polarization were also studied.

We believe this is the first systematic step in this type of approaches in quantifying Twitter users' behavior. The
Retweet matrix and retweet average scores can be used to develop new models and algorithms to analyze more complex tweet-and-retweet features. Our optimization framework can readily be adapted to account to incorporate other types of information. For example:

- The $\mathbf{y}$ vector does not need to be computed from sentiment analysis of tweets. It can be built from exogenous information that can be used to match the opinions of the Twitter retweet population, such as poll results.

- The A matrix is currently built with each row corresponding to one event, but the correspondence can be made with respect to other groupings of tweets and retweets, such as by eco- 


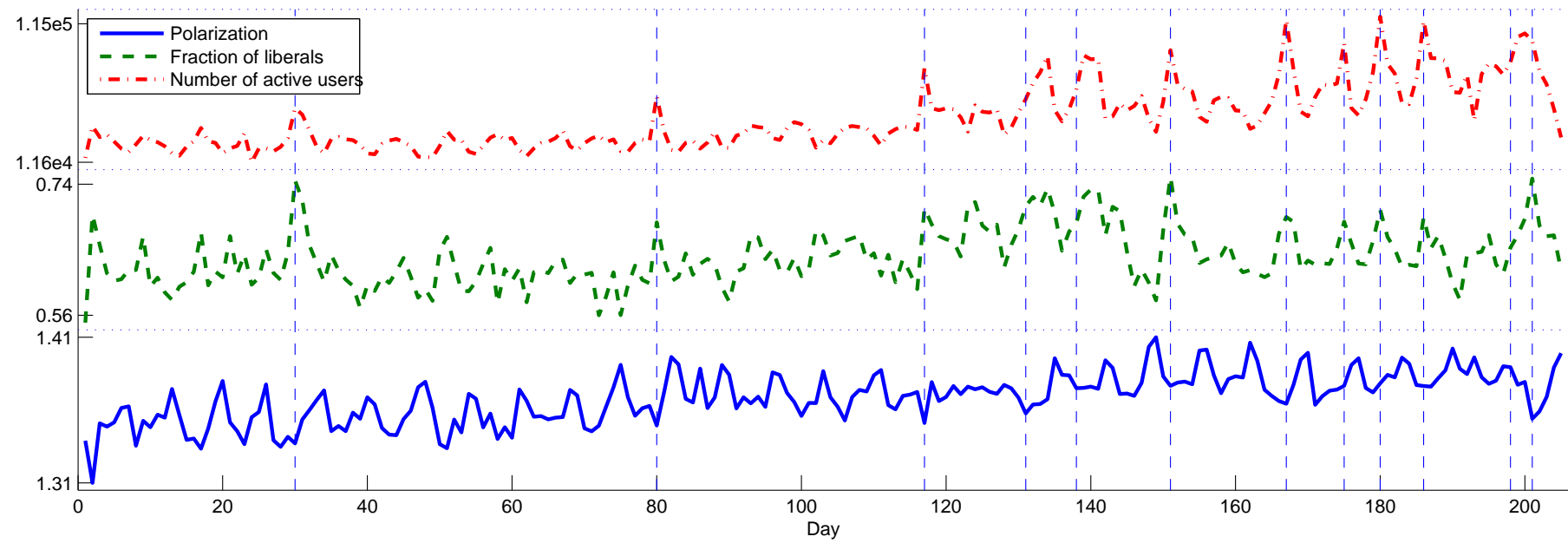

Fig. 13. Skew measures of ordinary users across time. Days with significant data loss during data collection are omitted. Liberal fraction and polarization are negatively correlated during events.

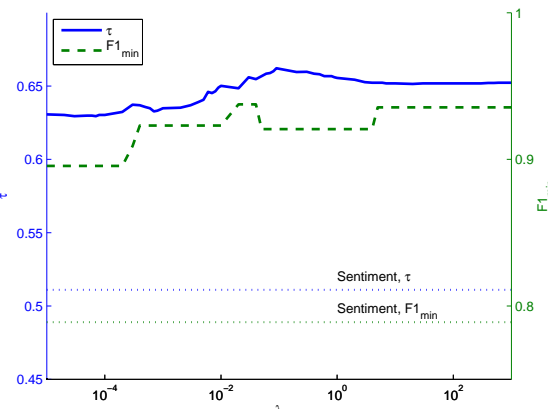

(a) Performance w.r.t. overall regularization (b) strength.

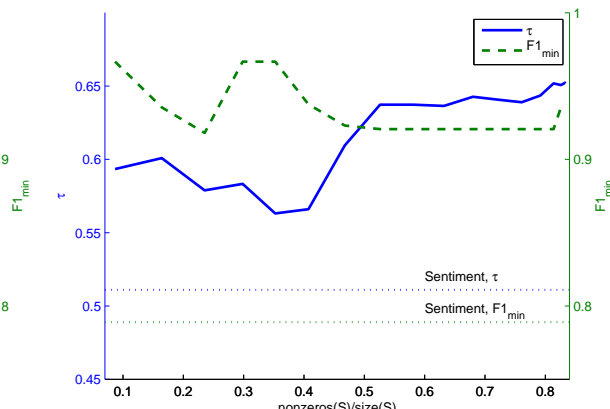

(b) Pertian.
cian.

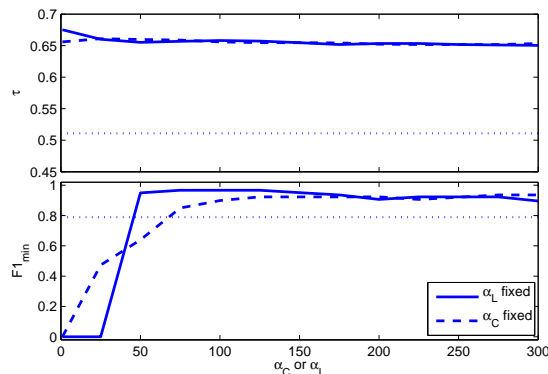

(c) Performance w.r.t. anchors' regularization strength.

Fig. 14. Our inference technique is robust with respect to parameter variations.

nomic/diplomatic/religious issues.

- The $\mathbf{W}$ matrix can be constructed from other types of network data (e.g., the network of mentions) or similarity measures.

- Our methodology is also applicable to other OSNs with retweet-like endorsement mechanisms, such as Facebook and YouTube with "like" functionality.

\section{ACKNOWLEDGMENTS}

This work was in part supported by ARO W911NF-11-10036, NSF NetSE CNS-0905086, and the Research Grants Council of Hong Kong under Project No. RGC CityU 125212. We also thank Prof. John C.S. Lui for fruitful discussions.

\section{REFERENCES}

[1] L. A. Adamic and N. Glance, "The political blogosphere and the 2004 U.S. election: Divided they blog," in Proc. LinkKDD, 2005.

[2] F. Al Zamal, W. Liu, and D. Ruths, "Homophily and latent attribute inference: Inferring latent attributes of Twitter users from neighbors," in Proc. ICWSM, 2012.

[3] J. An, M. Cha, K. P. Gummadi, J. Crowcroft, and D. Quercia, "Visualizing media bias through Twitter," in Proc. ICWSM SocMedNews Workshop, 2012.

[4] S. Ansolabehere, R. Lessem, and J. M. Snyder, "The orientation of newspaper endorsements in U.S. elections,"
Quarterly Journal of Political Science, vol. 1, no. 4, pp. 393404, 2006.

[5] P. Barberá, "Birds of the same feather tweet together: Bayesian ideal point estimation using Twitter data," $\mathrm{Po}$ litical Analysis, 2014.

[6] A. Boutet, H. Kim, and E. Yoneki, "What's in your tweets? I know who you supported in the UK 2010 general election," in Proc. ICWSM, 2012.

[7] S. Boyd and L. Vandenberghe, Convex Optimization. Cambridge University Press, 2004.

[8] J. Clinton, S. Jackman, and D. Rivers, "The statistical analysis of roll call data," American Political Science Review, vol. 98, no. 2, pp. 355-370, 2004.

[9] R. Cohen and D. Ruths, "Classifying political orientation on Twitter: It's not easy!" in Proc. ICWSM, 2013.

[10] M. D. Conover, B. Gonçalves, J. Ratkiewicz, A. Flammini, and F. Menczer, "Predicting the political alignment of Twitter users," in Proc. IEEE SocialCom, 2011.

[11] M. D. Conover, J. Ratkiewicz, M. Francisco, B. Gonçalves, A. Flammini, and F. Menczer, "Political polarization on Twitter," in Proc. ICWSM, 2011.

[12] M. Fiedler, "A property of eigenvectors of nonnegative symmetric matrices and its application to graph theory," Czechoslovak Mathematical Journal, vol. 25, no. 4, pp. 619633, 1975.

[13] S. Finn, E. Mustafaraj, and P. T. Metaxas, "The coretweeted network and its applications for measuring the perceived political polarization," in Proc. WEBIST, 2014.

[14] J. L. Fleiss, "Measuring nominal scale agreement among 
many raters," Psychological Bulletin, vol. 76, no. 5, pp. 378382, 1971.

[15] M. Gabielkov, A. Rao, and A. Legout, "Studying social networks at scale: Macroscopic anatomy of the Twitter social graph," in Proc. SIGMETRICS, 2014.

[16] M. Gentzkow and J. M. Shapiro, "What drives media slant? Evidence from U.S. daily newspapers," Econometrica, vol. 78, no. 1, pp. 35-71, January 2010.

[17] S. Gerrish and D. Blei, "How the vote: Issue-adjusted models of legislative behavior," in Proc. NIPS, 2012.

[18] - "Predicting legislative roll calls from text," in Proc. ICML, 2011.

[19] J. Golbeck and D. Hansen, "A method for computing political preference among Twitter followers," Social Networks, vol. 36, pp. 177-184, 2014.

[20] T. Groseclose and J. Milyo, "A measure of media bias," The Quarterly Journal of Economics, vol. 120, no. 4, pp. 11911237, November 2005.

[21] D. E. Ho and K. M. Quinn, "Measuring explicit political positions of media," Quarterly Journal of Political Science, vol. 3, no. 4, pp. 353-377, 2008.

[22] A. S. King, F. J. Orlando, and D. B. Sparks, "Ideological extremity and primary success: A social network approach," in Proc. MPSA Conference, 2011.

[23] J. R. Landis and G. G. Koch, "The measurement of observer agreement for categorical data," Biometrics, vol. 33, no. 1, pp. 159-174, 1977.

[24] Y.-R. Lin, J. P. Bagrow, and D. Lazer, "More voices than ever? quantifying media bias in networks," in Proc. ICWSM, 2011.

[25] A. Livne, M. P. Simmons, E. Adar, and L. A. Adamic, "The party is over here: Structure and content in the 2010 election," in Proc. ICWSM, 2011.

[26] J. R. Lott and K. A. Hassett, "Is newspaper coverage of economic events politically biased?" Online, October 2004, http:/ /dx.doi.org/10.2139/ssrn.588453.

[27] C. Lumezanu, N. Feamster, and H. Klein, "\#bias: Measuring the tweeting behavior of propagandists," in Proc. ICWSM, 2012.

[28] Y. Mejova, P. Srinivasan, and B. Boynton, "GOP primary season on twitter: "Popular" political sentiment in social media," in Proc. WSDM, 2013.

[29] P. T. Metaxas and E. Mustafaraj, "Social media and the elections," Science, vol. 338, pp. 472-473, 2012.

[30] S. A. Munson, S. Y. Lee, and P. Resnick, "Encouraging reading of diverse political viewpoints with a browser widget," in Proc. ICWSM, 2013.

[31] E. Mustafaraj, S. Finn, C. Whitlock, and P. T. Metaxas, "Vocal minority versus silent majority: Discovering the opinions of the long tail," in Proc. SocialCom/PASSAT, 2011.

[32] S. Park, M. Ko, J. Kim, Y. Liu, and J. Song, "The politics of comments: Predicting political orientation of news stories with commenters' sentiment patterns," in Proc. CSCW, 2011.

[33] M. Pennacchiotti and A.-M. Popescu, "A machine learning approach to Twitter user classification," in Proc. ICWSM, 2011.

[34] K. T. Poole and H. Rosenthal, "A spatial model for legislative roll call analysis," American Journal of Political Science, vol. 29, no. 2, pp. 357-384, 1985.

[35] E. Seneta, Non-negative Matrices and Markov Chains. Springer, 2007.

[36] M. Thelwall, K. Buckley, G. Paltoglou, D. Cai, and K. A., "Sentiment strength detection in short informal text," Journal of the American Society for Information Science and Technology, vol. 61, no. 12, pp. 2544-2558, 2010.

[37] A. Tumasjan, T. O. Sprenger, P. G. Sandner, and I. M. Welpe, "Predicting elections with Twitter: What 140 characters reveal about political sentiment," in Proc. ICWSM, 2010 .
[38] S. Volkova, G. Coppersmith, and B. Van Durme, "Inferring user political preferences from streaming communications," in Proc. ACL, 2014.

[39] J. Wang, W. X. Zhao, Y. He, and X. Li, "Infer user interests via link structure regularization," ACM Transactions on Intelligent Systems and Technology, vol. 5, no. 2, 2014.

[40] I. Weber, V. R. K. Garimella, and E. Borra, "Mining web query logs to analyze political issues," in Proc. WebSci, 2012.

[41] I. Weber, V. R. K. Garimella, and A. Teka, "Political hashtag trends," in Proc. ECIR, 2013.

[42] F. M. F. Wong, C. W. Tan, S. Sen, and M. Chiang, "Quantifying political leaning from tweets and retweets," in Proc. ICWSM, 2013.

[43] D. X. Zhou, P. Resnick, and Q. Mei, "Classifying the political leaning of news articles and users from user votes," in Proc. ICWSM, 2011. 